Original Articles

\title{
High Serum Erythropoietin and Ferritin Levels in Conjunction with Anemia Response in Malignant Lymphoma
}

\author{
Sofia Omari ${ }^{1,2}$, Alhossain Khalafallah ${ }^{1,3}$, Mahmoud Ayesh $^{4}$, Ismail Matalka $^{2}$ and Raji Al-Hadithi ${ }^{4}$ \\ ${ }^{1}$ School of Human Life Sciences, University of Tasmania, Newnham, Tasmania, 7248, Australia, \\ ${ }^{2}$ Department of Pathology, Pathology \& Laboratory Medicine Department, Jordan University of Science and \\ Technology, King Abdullah University Teaching Hospital, Irbid, Jordan. \\ ${ }^{3}$ Department of Haematology, Launceston General Hospital, Tasmania, Australia. \\ ${ }^{4}$ Department of Internal Medicine, Jordan University of Science and Technology, Irbid, Jordan.
}

Correspondence to: A/Professor A. Khalafallah, Department of Haematology, Launceston General Hospital, Tasmania, Australia. E-mail Khalafallah@,dhhs.tas.gov.au

Competing interests: The authors have declared that no competing interests exist.

Published: May 16, 2011

Received: January 18, 2011

Accepted: April 10, 2011

Mediterr J Hematol Infect Dis 2011, 3: e2011018, DOI 10.4084/MJHID.2011.018

This article is available from: http://www.mjhid.org/article/view/7867

This is an Open Access article distributed under the terms of the Creative Commons Attribution License (http://creativecommons.org/licenses/by/2.0), which permits unrestricted use, distribution, and reproduction in any medium, provided the original work is properly cited.

Abstract: Anemia is a common finding in lymphoma. There are few data available regarding the erythropoietin (EPO) levels in conjunction with ferritin in lymphoma patients. We prospectively evaluated 55 patients diagnosed with malignant lymphoma during the period between November 2006 and March 2008 at the King Abdullah University Teaching Hospital, Jordan. Our data showed that $74.4 \%$ of lymphoma patients were anemic. Furthermore, serum EPO and ferritin levels were higher in lymphoma patients compared with the healthy controls $(P=0.001)$. The observed versus predicted EPO ratio showed also significantly higher levels in anemic lymphoma patients compared to healthy controls $(p=0.03)$. There was an improvement in the Hb level in lymphoma patients who were treated with at least 3-cycles of chemotherapy as compared with newly-diagnosed patients. An adequate increase of EPO levels was observed in anemic lymphoma patients and notably associated with higher ferritin levels and improvement of $\mathrm{Hb}(\mathbf{p}<0.001)$.

Our findings suggest that ferritin estimation in lymphoma patients may predict the level of erythropoiesis and possibly the degree of anemia. Further studies to confirm these findings are warranted.

Introduction: Anemia is common in patients with cancer, especially Hodgkin's lymphoma (HL) and nonHodgkin's lymphomas (NHL). ${ }^{1,2}$ About 30-40\% of lymphoma patients present with anemia prior to commencement of chemotherapy. The anemia is mainly attributed to bone marrow replacement by lymphoma cells or inadequate erythropoietin (EPO) production which leads to suppressed erythropoiesis. ${ }^{3}$ The frequency of anemia increases in all malignancies, with the largest increase in patients with NHL (from $35.1 \%$ at baseline to $73.7 \%$ ) and $\mathrm{HL}$ (from $21.9 \%$ to $54.5 \%$ ) under the age of $60 .^{4-6}$ EPO stimulates the 
proliferation and maturation of erythroid precursors in the bone marrow. The earliest progenitor form that can be identified is the erythroid burst-forming unit (BFUE), which subsequently evolve into erythroid colonyforming units (CFU-E). EPO acts primarily on these cells by stimulating cell division and maturation as well as inhibiting apoptosis. ${ }^{7}$ Tissue hypoxia triggers endogenous EPO production when hemoglobin $(\mathrm{Hb})$ falls below $12 \mathrm{~g} / \mathrm{dL}^{8,9}$ When hypoxia resolves, EPO production decreases. ${ }^{9}$

Initial studies in patients with cancer found that most of the patients had disproportionately low levels of endogenous EPO for the degree of their anemia, especially after chemotherapy. ${ }^{10-12}$ Some studies showed that cancer-related anemia patients had serum EPO levels above the normal range which shows their strong ability into self-body regulation in response to anemia. ${ }^{10-12}$ Although some studies suggested that EPO levels decrease in malignant lymphoma, ${ }^{11,13}$ however, others reported that EPO levels increase in anemic lymphoma patients, indicating that anemia did not depend on defective EPO secretion. ${ }^{14}$ Ferritin is synthesised and released from malignant lymphocytes in a faster manner than normal lymphocytes causing ferritin concentration to increase in those patients. ${ }^{15-17}$ However, there is very few studies that address EPO response in association with ferritin levels in conjunction with anemia response in malignant lymphoma. The aims of our study were to determine (a) the EPO and ferritin levels in de novo and treated lymphoma patients, and (b) whether there is a relationship between EPO level and ferritin response to anemia.

Patients and Methods: Fifty-five serum samples were obtained from patients who were diagnosed with malignant lymphomas (29 HL and $26 \mathrm{NHL}$ ) during the period between November 2006 to March 2008 at the King Abdullah University Teaching Hospital. Written consent was obtained from all patients. This study was ethically approved by the Conjoint Ethics Committee of Jordan University of Science and Technology. Thirty samples were obtained from newly-diagnosed patients and twenty-five from patients who were receiving chemotherapy after at least 3 cycles of chemotherapy. Both groups included anemic and nonanemic patients. The specific diagnosis and the stage of the disease for each lymphoma patient were obtained from patients clinical notes. We included in the study newly diagnosed patients with malignant lymphoma and patients who had received at least three cycles of chemotherapy for malignant lymphoma and had not received blood transfusions for at least 12 weeks.

There were an equal number of samples collected at the time of diagnosis and after 3 cycles of chemotherapy. Bone marrow studies were performed for all patients and showed that 13 cases had bone marrow involvement (stage IV lymphoma disease) without a significant compromise of the hemopoeitic reserve.

We excluded patients with hepatitis, infections, liver disease, renal failure, iron, B12 and folate deficiencies and patients with hemolytic anemia as these conditions may influence the levels of ferritin and EPO. Infections markers were negative for the patients included in the study. Furthermore, no patients received erythropoietin therapy in this study.

Fifty-five healthy, sex and age-matched volunteers were randomly selected as a control group. All control group participants provided a written consent.

Non-fasting 5-10 $\mathrm{ml}$ serum samples were obtained from all participants during the morning at room temperature. Samples were centrifuged within 30 minutes of collection at $2000 \mathrm{rpm}$ for 10 minutes at room temperature and sera were isolated from each sample. For each patient, the following tests were performed: serum EPO, $\mathrm{Hb}$, creatinine, lactate dehydrogenase (LDH), B12, folate and ferritin. Serum vitamin B12 was obtained to exclude megaloblastic anemia. EPO was measured by ELISA (IBL-Hamburg kit, Germany). Ferritin levels were measured by electrochemiluminescence immunoassay (ECLIA) using an Elecsys 1020-immunoassay analyzer (Roche Diagnostics, Indianapolis, Indiana, USA).

Definitions: Stage A lymphoma disease is defined by absence of constitutional symptoms, while stage B means presence of B-constitutional symptoms such as weight loss, night sweats or fever.

Stage I-II lymphoma represent a localized nodal disease, while stage III lymphoma disease represents wide-speared nodal disease but with absence of nonreticuloendothelial organ involvement, and stage IV lymphoma disease reflects organ involvement such as bone marrow, liver or lung involvement. Extra-nodal disease is defined as involvement of other organs that are not related to the reticulo-endothelial system. Severe anemia is defined by a level of $\mathrm{Hb}<8 \mathrm{~g} / \mathrm{dl}$, while moderate anemia; $\mathrm{Hb}=8-10 \mathrm{~g} / \mathrm{dl}$ and mild anemia in males; $\mathrm{Hb}=10-13 \mathrm{~g} / \mathrm{dl}$ and in females; $\mathrm{Hb}=10-12$ $\mathrm{g} / \mathrm{dl}$.

Statistical analysis: Means, standard deviations (SD) and difference of means were estimated for illustrative purposes using general linear modelling. Assumptions of linear regression were violated in most of these analyses due to heteroskedasticity and skewness of residuals; therefore, non-parametric analyses (ordinal logistic regression) were performed to test differences of distributions. P-values were corrected for multiple 
Table 1. Characteristics and laboratory results of the patients and healthy controls

\begin{tabular}{|c|c|c|c|c|c|c|}
\hline & & Healthy controls & Lymphoma patients & & Comparison & \\
\hline & & Mean (SD) & Mean (SD) & Difference & $95 \%$ CI & P-value \\
\hline Age (years) & & $43.6(1.9)$ & $40.7(14.2)$ & -2.9 & $(-6.8$ to 1.0$)$ & 0.14 \\
\hline $\begin{array}{l}\text { EPO level } \\
\text { (mU/I) }\end{array}$ & $3-20$ & $7.0(5.3)$ & $26.2(25.8)$ & 19.2 & (12.2 to 26.2$)$ & $<0.001$ \\
\hline $\begin{array}{ll}\text { O/P } & \text { EPO } \\
\text { ratio } & \end{array}$ & & $1.70(2.00)$ & $1.04(1.29)$ & -0.66 & $(-1.29$ to -0.04$)$ & 0.038 \\
\hline Hb (g/dl) & $\begin{array}{c}14-18 \text { (male) } \\
12-16 \text { (female) }\end{array}$ & $13.7(1.4)$ & $11.0(1.9)$ & -2.7 & $(-3.3$ to -2.1$)$ & $<0.001$ \\
\hline $\begin{array}{l}\text { Ferritin } \\
(\mathrm{ng} / \mathrm{ml})\end{array}$ & $20-300$ & $43(24)$ & $627(729)$ & 583 & (359 to 808$)$ & $<0.001$ \\
\hline
\end{tabular}

EPO, serum erythropoietin. Hb, hemoglobin. Mean (SD), and difference estimated by general linear modeling for illustrative purposes, and comparison tested using Mann-Whitney rank-order test due to non-normal distributions. O/P, observed/predicted EPO ratio after adjustment for endogenous EPO response from the normal EPO production/activity.

comparisons where appropriate by the Holm method. All statistical analyses were performed using Stata/SE 11.0 (StataCorp, College Station, Tx USA).

Observed/Predicted (O/P) EPO ratio was calculated in order to determine the endogenous erythropoietin response to the level of $\mathrm{Hb}$ in our cohort of patients while at the same time avoiding possible interference by the endogenous erythropoietin production and/or erythropoietin activity.

The O/P EPO ratio has been calculated by dividing the observed log erythropoietin levels of the patients by the predicted log erythropoietin levels to obtain the $\mathrm{O} / \mathrm{P}$ ratio. The same ratio has been also calculated for the control group.

Results: We estimated serum EPO levels in 55 normal healthy controls where the EPO mean was $7 \mathrm{mU} / \mathrm{L}$ $(\mathrm{SD} \pm$ 5.3). We observed that lymphoma patients had higher EPO levels compared with the healthy control group. $\mathrm{Hb}$ was significantly lower in lymphoma patients compared with the control group $(\mathrm{P}<0.001)$. Lymphoma patients had significantly higher ferritin levels than the control group $(\mathrm{P}<0.001$; Table 1). Also, $\mathrm{Hb}$ was lower in newly-diagnosed lymphoma patients when compared to the lymphoma patients in the chemotherapy group $(\mathrm{P}=0.003)$. $\mathrm{EPO}, \mathrm{LDH}$ and ferritin concentrations were similar for newly-diagnosed lymphoma patients and patients who underwent chemotherapy. Stage B lymphoma (symptomatic) patients comprised most of the lymphoma study group (73.9 \%) compared with $19.6 \%$ for stage A lymphoma (asymptomatic) and 6.5\% for extranodal involvement patients. Stage A lymphoma patients had significantly higher $\mathrm{Hb}$ concentrations compared with those with stage $\mathrm{B}(\mathrm{P}=0.028)$. However, EPO levels were not significantly higher in stage $\mathrm{B}$ lymphoma patients compared with stage A patients $(\mathrm{P}=0.4)$, and there were no significant differences observed in $\mathrm{LDH}$ and ferritin levels between stage A and stage B lymphoma patients. There was no statistical difference seen between HL and NHL patients in regard to $\mathrm{EPO}, \mathrm{Hb}$, ferritin, and LDH levels.

Anemic patients accounted for $74.4 \%$ of the sample patients while non-anemic patients for $25.6 \%$. There were 4 cases who had severe anemia and 19 had moderate anemia and 18 had mild anemia, while 14 patients were not anemic.

Anemic patients had higher levels of EPO than non-anemic patients or healthy controls $(\mathrm{P}=0.001)$. However, the observed/predicted EPO ratio after adjustment for endogenous EPO response from the normal EPO production/activity continue to demonstrate significantly higher levels in anemic lymphoma patients versus healthy controls $(\mathrm{p}=0.03)$ (Table 1). There were significantly higher ferritin levels in non-anemic lymphoma patients compared with the healthy control group ( $\mathrm{P}=0.015$; Figure 1). $\mathrm{EPO}, \mathrm{Hb}$ and ferritin levels were significantly higher in non-anemic lymphoma patients compared to healthy controls.

$\mathrm{Hb}$ levels were significantly lower in "high EPO group" compared to the "low-normal EPO group".

Discussion: Our analysis showed that serum EPO levels were higher in lymphoma patients compared with the healthy control group. This is most likely due to EPO response to anemia in lymphoma patients. Furthermore, anemic lymphoma patients $(74.4 \%)$ had higher EPO levels compared to non-anemic lymphoma patients. Similar findings have been reported by Birgegård et $a l ;^{5} \mathrm{Li}$ et $a l^{12}$ and others. ${ }^{18}$ Serum EPO concentrations in lymphoma patients exhibited wide variation, ranging from normal levels to high levels in association with anemia. Some studies showed a decreased level of EPO in treated lymphoma patients, ${ }^{3,11,13}$ whereas the present study demonstrats no 


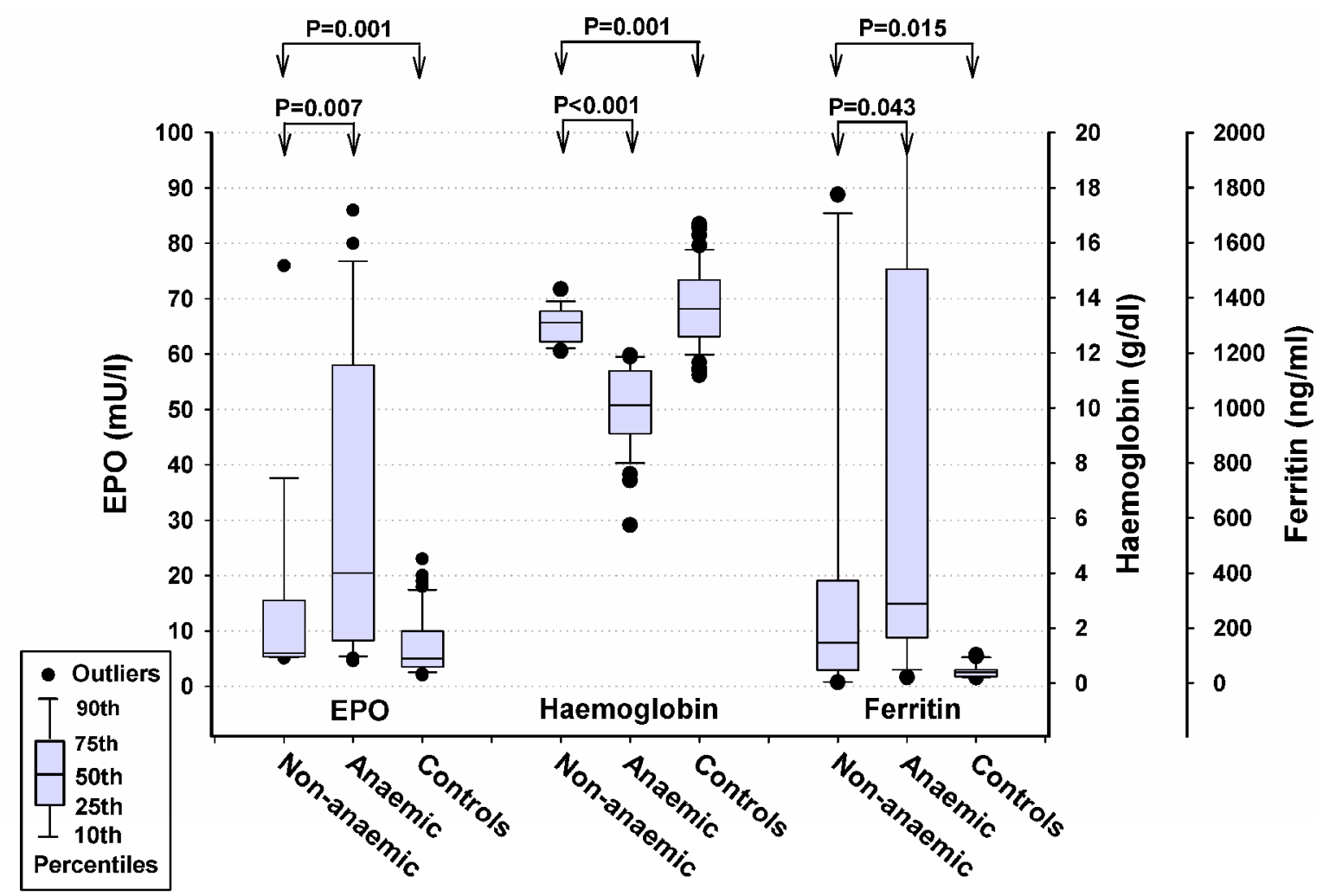

Figure 1. Distributions of non-anemic and anemic lymphoma patients compared with normal healthy controls

difference in EPO levels between newly-diagnosed lymphoma patients and patients on chemotherapy. Our results are consistent with a previous study reported by Ozguroglu et al. ${ }^{18}$ However, Lee ${ }^{11}$ reported low levels of EPO in patients receiving chemotherapy compared with newly-diagnosed patients. An inverse relationship between $\mathrm{Hb}$ level and EPO was seen in our series in accordance with other studies. ${ }^{3,18}$ Furthermore, the current study showed an improvement in $\mathrm{Hb}$ level in lymphoma patients treated with chemotherapy for at least three cycles as compared with patients who were newly-diagnosed $(\mathrm{P}=0.003)$. This is largely due to the fact that most of the treated patients for lymphoma disease were in advanced clinical stage III-IV (65\%) that has responded with improvement of $\mathrm{Hb}$ secondary to the commenced treatment. Furthermore, stage A lymphoma patients had significantly higher initial $\mathrm{Hb}$ levels compared with stage $\mathrm{B}$ patients $(\mathrm{P}=0.028)$. Higher EPO levels were found in stage $B$ patients in comparison with stage A but was not statistically significant. We observed higher ferritin levels in lymphoma patients with high EPO levels compared to healthy controls $(\mathrm{P}<0.001)$, that has been explained by erythropoietic response to anemia. Therefore, the ferritin level may indicate the increase of EPO activity among lymphoma patients.

Conclusions: In summary, an adequate EPO and ferritin response to anemia in patients with lymphoma was demonstrated in our study. This may indicate that recombinant human EPO (rhuEPO) treatment is not required in anemic lymphoma patients who have acceptable EPO and ferritin response. Results obtained from this study would help in understanding different factors that contribute in the degree of anemia among lymphoma patients. Furthermore, most of the patients with high ferritin levels had significantly high EPO levels. Perhaps more studies can address utilization of ferritin as a possible marker for EPO activity in addition to measuring the response to anemia in this cohort of patients. Of note, the cost of ferritin measurement is much lower than that of EPO. Moreover, ferritin testing is more accessible in most laboratories compared to EPO. However, one of the important limitations is that ferritin level can be affected as an acute phase reactant by the degree of infection or inflammation that may coincide in lymphoma patients. It is worth noting that we excluded all patients with infections from the current study. Nevertheless, further studies to confirm our findings are warranted.

Acknowledgements: This work was supported by a grant from Jordan University of Science and Technology (J.U.S.T), Jordan. All thanks to the King Abdullah University Teaching Hospital which allowed access to diagnostic materials and pathology reports. 


\section{References:}

1. Smith Jr R, Tchekmedyian S. Practitioners' practical model for managing cancer-related anemia. Oncology (Williston Park, NY) 2002;16(S10):55-63.

2. Ludwig H, Strasser K. Symptomatology of anemia. Semin Oncol 2001;28(S8):7-14. doi:10.1016/S0093-7754(01)90206-4

3. Kostova G, Siljanovski N. Erythropoietin production in patients with malignant lymphoma. Prilozi 2005;26:157-68. PMid:16400237

4. Coiffier B. The impact and management of anaemia in haematological malignancies. Medical oncology 2000;17:S2-10.

5. Birgegård $G$, Gascón $P$, Ludwig H. Evaluation of anaemia in patients with multiple myeloma and lymphoma: findings of the European CANCER ANAEMIA SURVEY. European journal of haematology 2006;77:378-86. doi:10.1111/j.16000609.2006.00739.x PMid:17044835 PMCid:1618958

6. Steurer M, Wagner H, Gastl G. Prevalence and management of anaemia in haematologic cancer patients receiving cyclic nonplatinum chemotherapy: Results of a prospective national chart survey. Wiener Klinische Wochenschrift 2004;116:367-72. doi:10.1007/BF03040915 PMid:15291288

7. Sathyanarayana P, Dev A, Fang J, Houde E, Bogacheva O, Bogachev O, et al. EPO receptor circuits for primary erythroblast survival. Blood 2008; 11:5390-9. doi:10.1182/blood-2007-10-119743 $\quad$ PMid:18349318 PMCid:2396729

8. Gabrilove J. Overview: erythropoiesis, anemia, and the impact of erythropoietin. Semin Hematol 2000;37(S6):1-3. doi:10.1016/S0037-1963(00)90060-X

9. Haase VH. Hypoxic regulation of erythropoiesis and iron metabolism. American Journal of Physiology - Renal Physiology 2010; 299:1-13. $\quad$ doi:10.1152/ajprenal.00174.2010 PMid:20444740
10. Schwartz R. Anemia in patients with cancer: Incidence, causes, impact, management, and use of treatment guidelines and protocols. American Journal of Health-System Pharmacy 2007;64:5-13. doi:10.2146/ajhp060601 PMid:17244886

11. Lee S, Kwon J, Jung C. Erythropoietin response is inadequate in cancer patients receiving chemotherapy. International journal of hematology 2001;74:416-20. doi:10.1007/BF02982085 PMid:11794697

12. Li LB, Luo R. Clinical significance of serum erythropoietin detection in patients with cancer-related anemia. Di Yi Jun Yi Da Xue Xue Bao 2003;23:954-55. PMid:13129732

13. Han B, Shi Y, Zhu J, He X, Lin N, Li S, et al. Study on serum erythropoietin levels in patients with hematologic malignancies. Zhonghua xue ye xue za zhi 2006;27:543-45. PMid:17172129

14. Rokicka-Piotrowicz M, Paszkowska M, Kuratowska Z. Anemia and erythropoietin level in some hematology malignancies. Polskie archiwum medycyny wewn 1994;92:409-16.

15. Sarcione E, Smalley J, Lema M, Stutzman L. Increased ferritin synthesis and release by hodgkin's disease peripheral blood lymphocytes1. International Journal of Cancer 2006;20(3):33946.

16. Yildirim R, Gundogdu M, Erdem F, Kiki I, Bilici M. The Levels of Serum C-Reactive Protein, Beta 2 Microglobulin, Ferritin, Lactate Dehydrogenase and Some Specific Proteins in Patients with Non-Hodgkin's Lymphoma Before and After Treatment. The Eurasian Journal of Medicine 2009;41:165-68.

17. Knutson $\mathrm{M}$, Wessling-Resnick $\mathrm{M}$. Iron metabolism in the reticuloendothelial system. Crit Rev Biochem Mol Biol 2003;38:61-88. doi:10.1080/713609210 PMid:12641343

18. Ozguroglu M, Arun B, Demir G, Demirelli F, Mandel N, Buyukunal E, et al. Serum erythropoietin level in anemic cancer patients. Medical Oncology 2000;17:29-34. doi:10.1007/BF02826213 PMid:10713657 